\title{
Sleeping environment in Sudden Unexpected Infant Death cases: a population-based study in France
}

Levieux K. ${ }^{1}$, Patural H. ${ }^{2}$, Harrewijn I. ${ }^{3}$, Pidoux O. ${ }^{3}$, Briand Huchet E. ${ }^{4}$, Kugener B. ${ }^{5}$, de Visme S. ${ }^{6}$, Hanf M. ${ }^{6}$, Chalumeau M. ${ }^{7}$, Gras Le Guen C. ${ }^{1}$ and OMIN Study Group ${ }^{8}$

1 Pediatric Intensive Care Unit, Nantes University Hospital, Nantes, France, 2 Pediatric Intensive Care Unit, Saint-Étienne University Hospital, Saint Etienne, France, Pediatric Intensive Care Unit, Montpellier University Hospital, Montpellier, France, 4 Pediatric Intensive Care Unit, Antoine Béclère University Hospital, AP-HP,

Clamart, France, 5 Department of pediatric medicine, HFME Lyon, Bron, France, 6 National Institute of Health and Medical Research CIC1413, Nantes University Hospital, Nantes, France, 7 Paris Descartes University, Paris, France, 8 the French National SUID registry (Observatoire national des Morts Inattendues du Nourrisson, France).

\section{BACKGROUND AND AIMS}

Context: Sudden unexpected infant death (SUID) remains the leading cause of postneonatal mortality in developed countries despite preventive campaigns targeting its modifiable risk factors since the 1990s. Suboptimal implementation of the recommendations for a safe infant sleeping environment (SISE) has been suggested as a cause of avoidable SUID. No national data are available in France regarding the implementation of SISE recommendations.

Objective: Describe sleep environment characteristics of infants who died from SUID in France.

\section{METHODS}

We used data from the recently launched French national SUID registry (Observatoire national des Morts Inattendues du Nourrisson, OMIN), which collects data for all infants younger than 1 year old admitted for SUID in one of the 35 French referral centers since 2015. Mobile intensive care teams, pathologists and physicians of the referral centers prospectively collect data regarding socio-environmental and parental characteristics; medical history; clinical, radiological and autopsy findings; and cause assigned to death after a thorough case investigation. We estimated the frequency of implementation of each recommendation of the American Academy of Pediatrics SISE endorsed by French authorities.

\section{RESULTS}

\section{Population}

Between May 2015 and September 2017, 363 cases of SUID were recorded, 264 included in the registry; complete data were available for 239 (76\%)
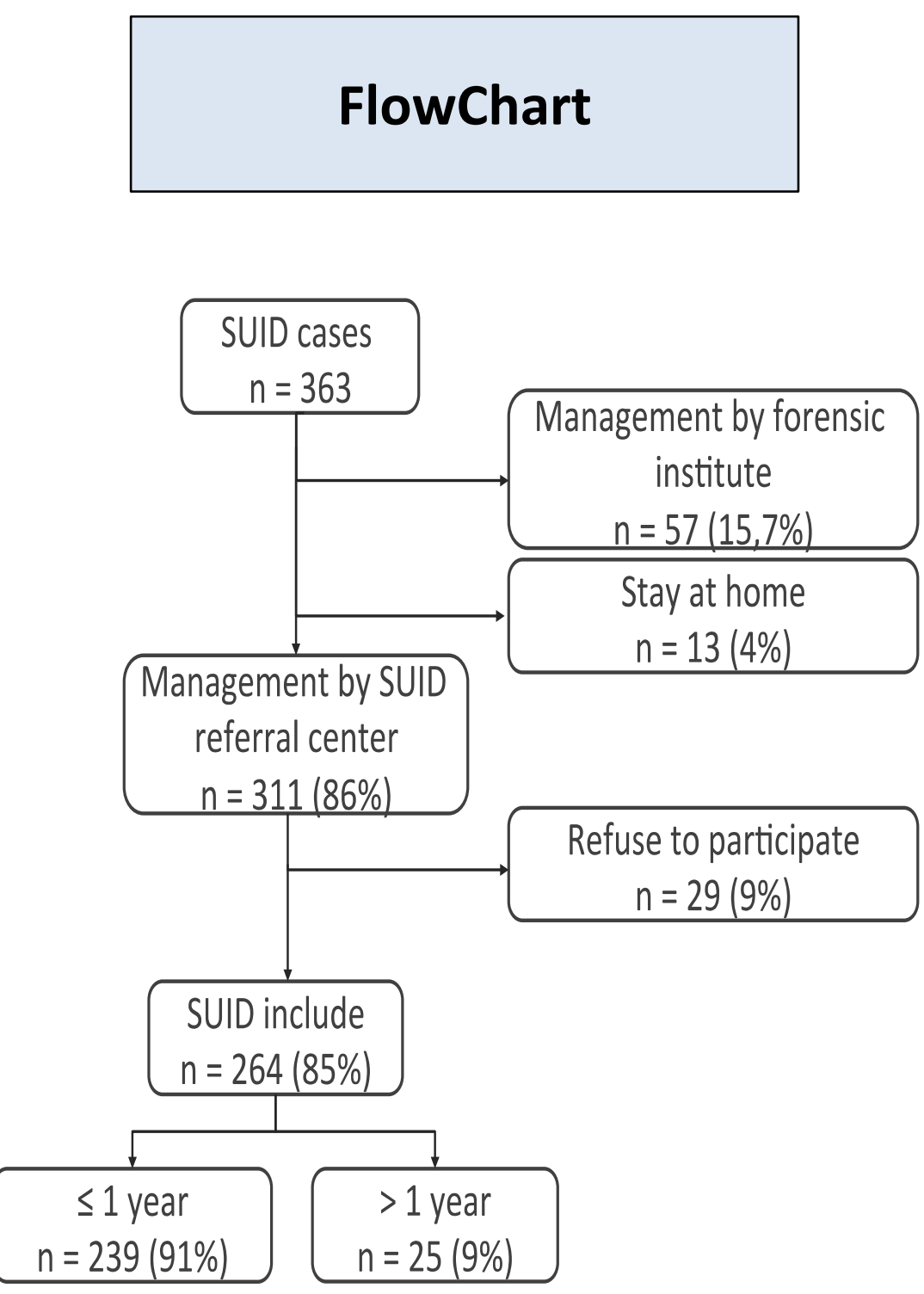

\section{Age at death (in month) $(n=264)$}

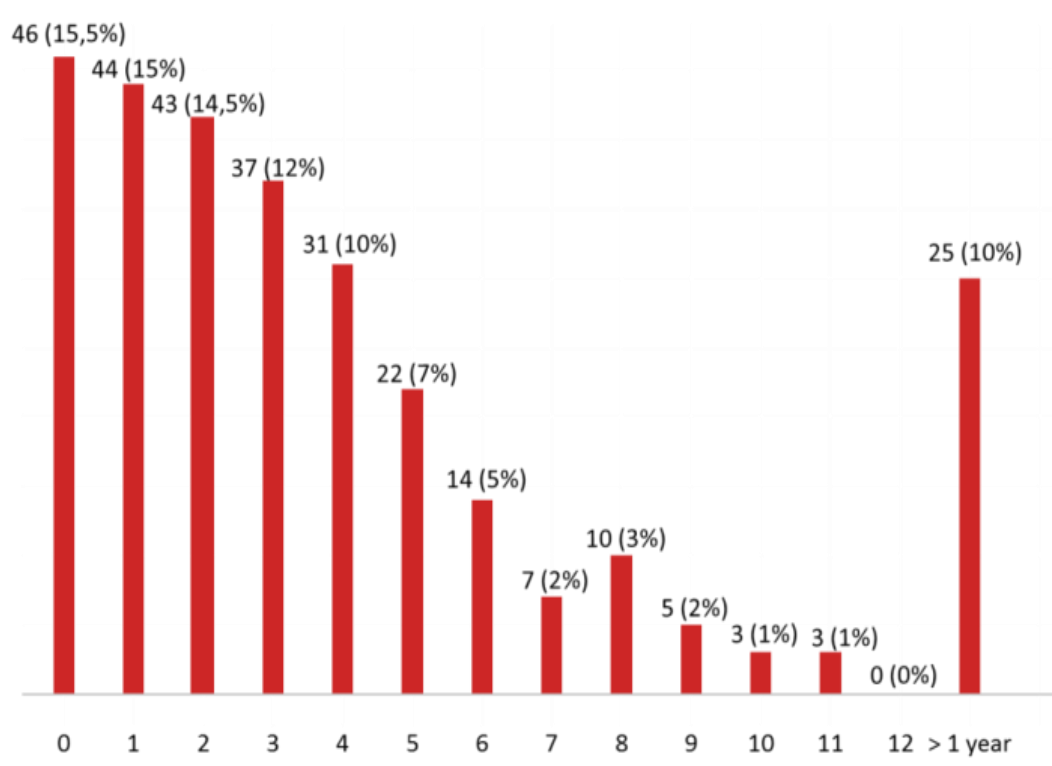

The median age of infants was 93 days, $91 \%$ were less than 1 year of age; and $16 \%$ were born preterm. $54 \%$ were male; $65 \%$ of SUID occurred at parent's home, $11 \%$ at childminder'home, $1 \%$ in nursery. At the time of death, the SUID risk factors were prone or side sleeping position (29\%); co-sleeping in an adult bed (18\%); sleeping on couches (2\%); lack of room-sharing (75\%); presence in the bed of a blanket (25\%), a pillow $(17 \%),>1$ stuffed animals (18\%) or a bumper pad attached to the crib (11\%); and tobacco smoke exposure (37\%). $35 \%$ were given a pacifier at bedtime, $40 \%$ were being breastfed.

\section{Etiological diagnosis after investigations}

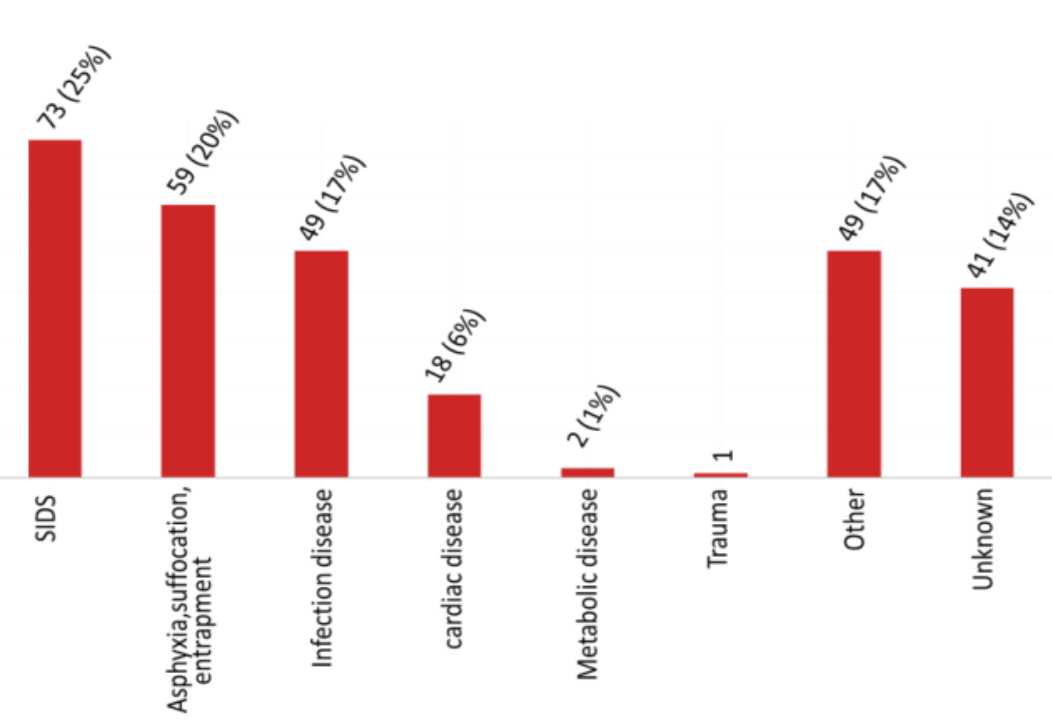

After usual causal investigations, $20 \%$ of cases, the assigned cause of death was suffocation, asphyxia, or entrapment; $17 \%$ to an infection, $6 \%$ to a cardiac and $1 \%$ to a metabolic disease and $17 \%$ to an other identified cause. $25 \%$ were classified as a Sudden Infant Death Syndrome (SIDS) due to a failure of postmortem examinations to identify any cause of death. A large heterogeneity in the management of SUID exists between the centers taking in charge SUID with regards to their investigations.

\section{CONCLUSION}

